\title{
Congruence of BOLD Response across Intertemporal Choice Conditions: Fictive and Real Money Gains and Losses
}

\author{
Warren K. Bickel, ${ }^{1}$ Jeffery A. Pitcock, ${ }^{1}$ Richard Yi, ${ }^{1}$ and Edgardo J. C. Angtuaco ${ }^{2}$ \\ ${ }^{1}$ Department of Psychiatry, Center for Addiction Research, Fred and Louis Dierks Research Laboratories, Psychiatric Research Institute and ${ }^{2}$ Department of \\ Radiology, University of Arkansas for Medical Sciences, Little Rock, Arkansas 72205
}

Intertemporal choice is predicated on the valuation of commodities with respect to delay until their receipt. Subjective value of a future outcome decreases, or is discounted, as a function of that delay (Bickel and Johnson, 2003). Although behavioral studies suggest no difference between the devaluation of real and fictive outcomes, no neuroimaging studies have investigated potential differences in the underlying deliberative process. Here, we compare behavioral and neural correlates of intertemporal valuation of real and hypothetical monetary gains as well as hypothetical losses, which have been posited to involve different mechanisms. Behavioral and neuroimaging sessions were conducted in which participants made intertemporal choice decisions in a gains condition using both real and hypothetical $\$ 100$ money and in a loss condition using a fictive \$100 money. Within-subject comparison of behavioral data revealed no significant difference between levels of discounting across the three conditions. Random-effects analysis of functional magnetic resonance imaging (fMRI) data of each of the three discounting conditions independently revealed significant signal change in limbic (anterior cingulate, striatum, posterior cingulate) and executive functioning areas (lateral prefrontal cortex), whereas a repeated-measures ANOVA failed to detect differences in signal change across the three discounting conditions after correcting for multiple comparisons. These data support a concordance between real and hypothetical conditions from delay-discounting studies and further suggest a congruence of the fMRI blood oxygen level-dependent signal across brain regions associated with the deliberative process of different forms of intertemporal choice.

\section{Introduction}

Delay (or temporal) discounting refers to the devaluation of an outcome as a function of the time to the delivery of that outcome. Delay discounting has been proposed to underlie impulsive decision making (Ainslie and Haendel, 1983; Bickel and Marsch, 2001), with increased levels of discounting observed in drugdependent cohorts (Madden et al., 1997; Kirby et al., 1999; Coffey et al., 2003).

A variety of reward outcomes have been used in discounting experiments including money, compact disks, alcohol, and cigarettes (Odum and Rainaud, 2003; Estle et al., 2007; Charlton and Fantino, 2008). These rewards are typically hypothetical and used in lieu of real rewards for three reasons. First, real reward outcomes can be cost prohibitive because of the number of data points typically collected. Second, temporal delays used in this assessment often include delays making delivery of

Received Nov. 5, 2008; revised March 16, 2009; accepted March 27, 2009.

This work was funded by National Institute on Drug Abuse Grants R01 DA11692 and R01 DA024080, Wilbur Mills Chair Endowment, and in part by the Arkansas Biosciences Institute, a partnership of scientists from Arkansas Children's Hospital, Arkansas State University, the University of Arkansas, Division of Agriculture, the University of Arkansas, Fayetteville, and the University of Arkansas for Medical Sciences. The Arkansas Biosciences Institute is the major research component of the Tobacco Settlement Proceeds Act of 2000. We thank D. Lindquist for her efforts in the experimental design and imaging protocols, D. Cardwell and D. Kesterson for their assistance in the acquisition of the imaging data, R. Landes for his assistance in the statistical analysis of behavioral data, and Samuel McClure and P. R. Montague for comments on earlier versions of this manuscript.

Correspondence should be addressed to Warren K. Bickel, Center for Addiction Research, University of Arkansas for Medical Sciences, 4301 West Markham Street \#843, Little Rock, AR 72205. E-mail: wbickel@uams.edu.

DOI:10.1523/JNEUROSCI.5319-08.2009

Copyright $\odot 2009$ Society for Neuroscience $\quad 0270-6474 / 09 / 298839-08 \$ 15.00 / 0$ the reward problematic (Johnson and Bickel, 2002). Third, providing real monetary outcomes to those with addictive disease, e.g., would be ethically questionable (Madden et al., 1997). However, potentially real rewards have been used whereby temporal delays are abbreviated and one or more randomly selected choices are awarded as opposed to every choice. This method assumes the participant will consider each trial as though the outcome were real, given that each choice has the potential of being realized. Johnson and Bickel (2002) awarded participants on average, one reinforcer for every 89.9 choices, whereas Madden et al. (2004) awarded one for every 412, and in a second experiment, one for every 15 . In all instances, within-subject comparisons failed to show a difference in discounting between the real and hypothetical reward conditions.

Although the behavioral research using delay discounting of commodities typically employs rewards (or gains), the discounting of losses has been less studied but has been reported as being qualitatively similar but with gains discounted to a greater degree than losses of the same commodity and magnitude (Murphy et al., 2001; Baker et al., 2003; Yi et al., 2006). However, reported differences between gains and losses, referred to as the sign effect, have not been determined to be consistent across discounting conditions (Yi et al., 2006). This quantitative difference, when observed, has led some to speculate that different processes are involved in the discounting of rewards and losses (Estle et al., 2006).

Although previous findings indicate that both real and hy- 
pothetical reward outcomes, as well as gains and losses are discounted in a similar manner, there has been a paucity of research investigating possible differences in the neural correlates associated with these intertemporal choice conditions. Whether the qualitative similarities or quantitative differences in choice behavior is matched in brain activations is unknown. Given the expansion of functional brain imaging studies of choice (McClure et al., 2004, 2007; Boettiger et al., 2007; Kable and Glimcher, 2007; Monterosso et al., 2007; Wittmann et al., 2007; Hoffman et al., 2008), knowledge of these effects is necessary for appropriate interpretation of this growing area of research.

Here, we conduct behavioral and functional magnetic resonance imaging (fMRI) assessments of 30 participants to examine (1) possible within-subject difference in behavioral measures of delay discounting between real and hypothetical rewards, as well as gains and losses of $\$ 100$ money and (2) potential within-subject differences in the neural correlates of intertemporal choice behavior between these three discounting conditions.

\section{Materials and Methods}

Thirty right-handed participants ( 21 females) between the ages of 20 and 67 years (mean age, $47.1 \pm 4.9$ ) with an average education of 14.0 years $( \pm 0.8)$ were consented under an Institutional Review Board-approved protocol at the University of Arkansas for Medical Sciences. Participants indicating substance abuse/dependence (other than cigarettes) or significant psychiatric disorder were excluded from participation. Subjects completed delay-discounting conditions including real money gains (RMG), hypothetical money gains (HMG), and hypothetical money losses (HML) in a behavioral session before scanning with an average time between behavioral session and scanning session of $38 \mathrm{~d}( \pm 10.5)$. Two previous studies have exhibited stability in the discounting of rewards over 2 and 3 month spans, respectively (Ohmura et al., 2006; Takahashi et al., 2007), but to our knowledge the stability of the discounting of losses has not been reported.

Behavioral discounting conditions were counterbalanced across participants and presented on computer using a decreasing amount algorithm (Du et al., 2002). Trials were presented on a computer monitor with the immediate alternative above a horizontal line bisecting approximately the middle of the screen and the delayed alternative beneath. Participants were instructed to respond to their preferred alternative with a hand-held response pad similar to the one that would be subsequently used within the scanner. Seven indifference points (at delays of 1 , $7,30,180,365,1825,9125$ d) were determined in the HMG and HML conditions. Four indifference points (at delays of 1, 7, 30, $180 \mathrm{~d}$ ) were determined in the RMG condition because of pragmatic issues regarding delivery of extremely delayed rewards. In this condition, one of the choices that the participant made was selected at random, and the participant was paid the outcome of that choice (one reinforcer for 24 choices).

Three delay-discounting conditions, designed to represent the three outcomes of interest obtained in the behavioral session, were administered to each participant during the scanning session: RMG, HMG, and HML. Each of the three conditions included two types of trials: (1) discounting trials, where participants indicated their preference between immediate and delayed outcomes (e.g., "Receive $\$ 99.85$ now," "Receive $\$ 100.00$ in 1 week") and (2) control trials, where the participants responded to their preference between outcomes without a temporal component (e.g., "Receive \$99.85," "Receive $\$ 100.00 ")$. In all discounting trials, the delayed outcome was fixed at $\$ 100$ with delays of 1 week, 1 month, and 6 months, whereas the immediate outcome was varied systematically (see supplemental material for additional information and full listing of stimuli; supplemental Table S1, available at www.jneurosci.org as supplemental material). Control trial stimuli were created to include common task-related physiological requirements (e.g., visual perception, premotor, motor) to better identify brain regions associated with the deliberative process by contrasting choices made in the discounting trials to those made in the control trials. These control stimuli were based on the choices appearing in the discounting trials; however, one half of the delayed amounts $(\$ 100)$ were substituted with an amount equal to $150 \%$ of the smaller alternative (e.g., "Receive $\$ 99.85$," "Receive $\$ 149.78$ ") to ensure that a judgment was being made with respect to the amount of the outcome and not a simple response to the location of the $\$ 100$ amount.

In all, there were 56 unique trials per condition (28 discounting trials and 28 control trials). Each trial was visually presented with one choice appearing above a horizontal line bisecting approximately the center of the screen and the second choice appearing below. For discounting trials, immediate and delayed outcomes were counterbalanced with respect to their position on the screen (top/bottom), as were the larger and smaller amounts contained within the control trials. Participants were informed before scanning that they would be awarded the outcome chosen in one randomly selected discounting trial of the RMG condition and that choices that appeared without an explicit temporal component were experimental controls and would not be considered for selection (one reinforcer for 28 choices). The HMG condition was identical to the RMG condition in every facet except for the awarding of a randomly selected trial choice. HMG and HML conditions differed only in that the word "Receive" was replaced by the word "Lose" in both discounting and control trials. Before the first trial in each of the three conditions, an instruction screen was presented that notified the participant of which task was about to be administered and required the participant to push a response pad button to proceed.

The stimuli were visually presented to participants in the scanner using IFIS-SA (Intergrated Functional Imaging System, Invivo). Participants were instructed to respond to their preferred alternative via button push of an MRI compatible response pad: right index finger for choice appearing above the horizontal line and right thumb for choice appearing below the horizontal line. Stimulus blocks were comprised of 3-4 trials of a trial-type (i.e., discounting, control) with each block of trials alternating. Trials within each block terminated at button-push (or at a maximum of $6000 \mathrm{~ms}$ ) at which time a fixation point would appear for a varied duration (3000-5000 ms) to jitter stimulus onset within each block. Each block was followed by a $12 \mathrm{~s}$ rest period (fixation point). See supplemental Figure $\$ 8$, available at www.jneurosci.org as supplemental material, for schematic diagram of experimental design. Each functional scan (per condition) consisted of acquiring 190 volumes (including two dummy scans preceding stimulus onset of the first trial to allow the magnet to reach a steady state and subsequently removed before preprocessing and analysis of the data). If the participant completed all 56 trials before acquiring 190 volumes, the sequence of trials would repeat without interruption or notification. The average number of trials completed in the RMG condition was $70.6 \pm 1.7$, with HMG, $70.5 \pm 1.6$ and HML, $69.3 \pm 1.5$. Order of presentation of conditions was balanced over participants to control for novelty.

Imaging data were acquired on a Siemens $3 \mathrm{~T}$ Trio using a standard head coil. T1-weighted high resolution anatomical images were acquired using a magnetization-prepared rapid gradient-echo sequence. Wholebrain, blood oxygen level-dependent (BOLD)-weighted images were acquired as participants responded to choices in three discounting conditions using an echo-planar imaging (EPI) sequence (repetition time, $2940 \mathrm{~ms}$, echo time, $30 \mathrm{~ms}$, flip angle, $90^{\circ}$, 36 slices acquired at a thickness of $4 \mathrm{~mm}$ with a $1 \mathrm{~mm}$ gap, field of view, $22 \mathrm{~cm}, 64 \times 64$ acquisition matrix, $3.44 \mathrm{~mm} \times 3.44 \mathrm{~mm}$ in plane resolution).

Imaging data were adjusted for slice acquisition time, corrected for motion by realigning volumes from the first condition acquired (per subject) to the third EPI volume (given that the first two dummy scans were excluded from the preprocessing) and creating a mean motioncorrected volume which was then subsequently used as a reference in realigning volumes from the two remaining conditions. The motioncorrected volumes were then normalized to Montreal Neurological Institute (MNI) coordinates, resampled at $3 \mathrm{~mm}^{3}$ resolution, and spatially smoothed with an $8 \mathrm{~mm}$ full-width half-maximum Gaussian kernel using SPM2. Signal changes were modeled as delta functions 
temporally synchronized with the onset of each trial and convolved with a canonical hemodynamic response function. Two regressors were established for each condition's general linear model to represent discounting trials and control trials and subsequently used to detect differences in responses between the two. We excluded events from the analysis where the participant failed to respond within $6 \mathrm{~s}$ of stimulus onset. The resulting individual $t$ maps were then entered into a second level, random effects analysis to establish significant patterns of activation at the group level and controlled for multiple comparisons using a False Discovery Rate (FDR) of $p<0.05$. We subsequently used the random effects results in a repeated-measures ANOVA to detect main effect statistical differences between the three conditions, as well as paired $t$ tests, and in a correlation analysis using prescan discounting parameters as predictors. Nonlinear transformations of MNI coordinates to Talairach coordinates were conducted using a Matlab function [mni2tal (http://imaging.mrc-cbu.cam.ac.uk/ imaging/MniTalairach)]. Transformed coordinates for cluster maximum voxels were subsequently entered into the Talairach daemon database (http://www.talairach.org) to establish Talairach and Brodmann area labels.

\section{Results}

\section{Behavioral session}

In a single behavioral session before scanning, each subject completed a computerized delay-discounting procedure in three conditions (\$100 RMG, \$100 HMG, and \$100 HML) where the magnitude of a delayed outcome is held constant, whereas the magnitude of an immediate outcome is systematically adjusted. In each trial of this procedure, subjects indicated their preference between an immediate outcome and a delayed outcome. A point of equilibrium is established representing the individual's indifference between the immediate and delayed amounts. In each condition, indifference points were determined at several delays and fit to a simple hyperbolic model of discounting (Mazur, 1987) using SAS Software to estimate a single index of discounting for each condition.

$$
V_{\mathrm{d}}=\frac{1}{V+k D}
$$

$V_{\mathrm{d}}$ represents the discounted value at $D$ delay, $V$ is the undiscounted amount, and $k$ is the estimated discounting parameter. High values of $k$ indicate greater discounting, and low values of $k$ indicate less discounting. Because of the skewed distributions shown to be associated with the hyperbolic function estimate, the estimated $k$ value for each individual/condition was log transformed $(\ln (k))$ to permit use of parametric statistics in the analysis. To more accurately measure differences between conditional measures of discounting as estimated using the hyperbolic equation (1), indifference points for delays in the hypothetical conditions that were not also collected in the real money condition (i.e., 365, 1825, $9125 \mathrm{~d}$ ) were excluded. A repeated-measures ANOVA was used to detect within-subject differences between $\ln (k)$ values in the three discounting conditions and indicated no differences in levels of discounting between RMG $\overline{\ln (k)}=$ $-5.42 \pm 0.95, \mathrm{HMG} \overline{\ln (k)}=-5.61 \pm 0.86$, and HML $\overline{\ln (k)}=$ $-5.60 \pm 1.37 ; F_{(2,28)}=0.21, p=0.81$ (Fig. $1 A$ ). We further examined area under the curve (AUC) measures (an alternative index of discounting) to compare the within-subject levels of discounting between the three conditions because AUC allows for freedom from the theoretical framework of the hyperbolic discounting function and problems associated with estimated parameters (Myerson et al., 2001). Results of the analysis of discount rates were confirmed with repeated-measures ANOVA using area under the curve measures. For the RMG, HMG, and

\section{A Mean $\ln (k)$ Differences \& $95 \% \mathrm{Cl}$}

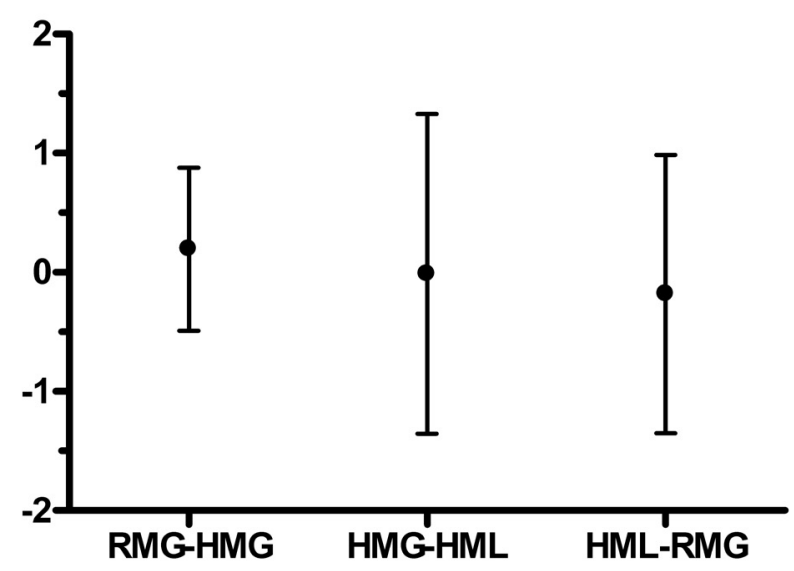

B Mean AUC Differences \& $95 \%$ Cl

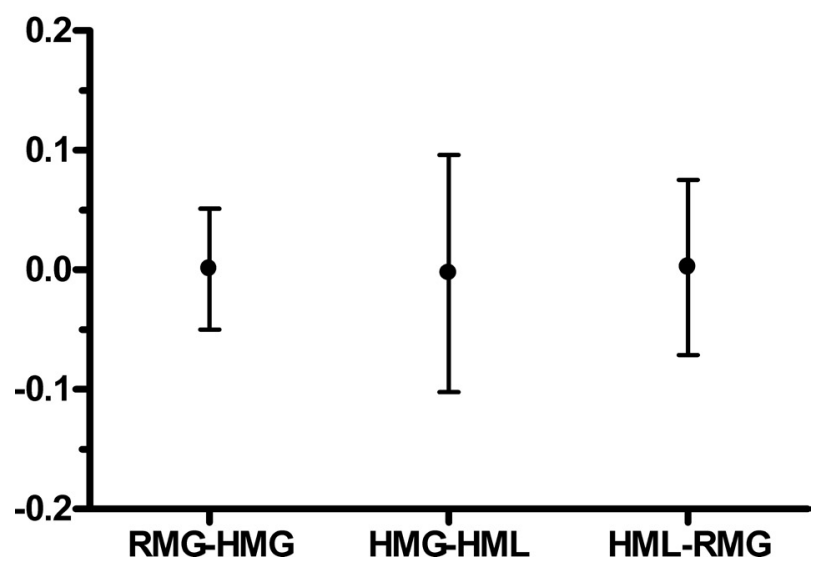

Figure 1. Indifference points for delays of 1, 7, 30, and $180 \mathrm{~d}$ were collected from each individual before scanning in each of three discounting conditions and were subsequently fitted to a hyperbolic curve using Mazur's (1987) nonlinear function. Estimated ks were then log transformed to address skewed distributions and then entered into a repeated-measures ANOVA to test for differences in discounting between the conditions. Within-subject differences between discounting conditions were determined not to be significant $\left(F_{(2,28)}=0.21, p=\right.$ 0.81): RMG-HMG $=0.196 \pm 0.68, \mathrm{HMG}-\mathrm{HML}=-0.014 \pm 1.34$, and $\mathrm{HML}-\mathrm{RMG}=$ $-0.18 \pm 1.17(\boldsymbol{A})$. Indifference points were also used to calculate AUC measures which were subsequently used in a repeated-measures ANOVA to detect within-subject difference between discounting conditions. AUC allows for freedom from the theoretical framework of the hyperbolic discounting function and problems associated with estimated parameters (Myerson et al., 2001). Results indicate no statistically significant difference between average normalized AUC measures $\left(F_{(2,28)}=0.00, p=0.99\right)(B):$ RMG $-\mathrm{HMG}=0.001 \pm 0.05, \mathrm{HMG}-\mathrm{HML}=$ $-0.003 \pm 0.10$, and $\mathrm{HML}-\mathrm{RMG}=0.002 \pm 0.07$.

HML conditions, $\overline{\mathrm{AUC}}$ was $0.72 \pm 0.09, \pm 0.10, \pm 0.10$, respectively $\left(F_{(2,28)}=0.00, p>0.99\right)$ (Fig. $\left.1 B\right)$. It should be noted that when $\ln (k)$ values for fictive gains and losses were estimated with the full number of indifference points, a significant difference between RMG and HMG was found (mean difference, $-0.93 ; t=$ -2.22 ; $\mathrm{df}=29 ; p=0.03$ ). In addition, we made no attempt to exclude any behavioral data from our analysis based on goodness of fit measures associated with the hyperbolic estimate of discounting $(k)$ as is frequently done.

\section{Imaging session}

Responses made to delay-discounting trials performed in the scanner were examined to investigate the consistency of dis- 


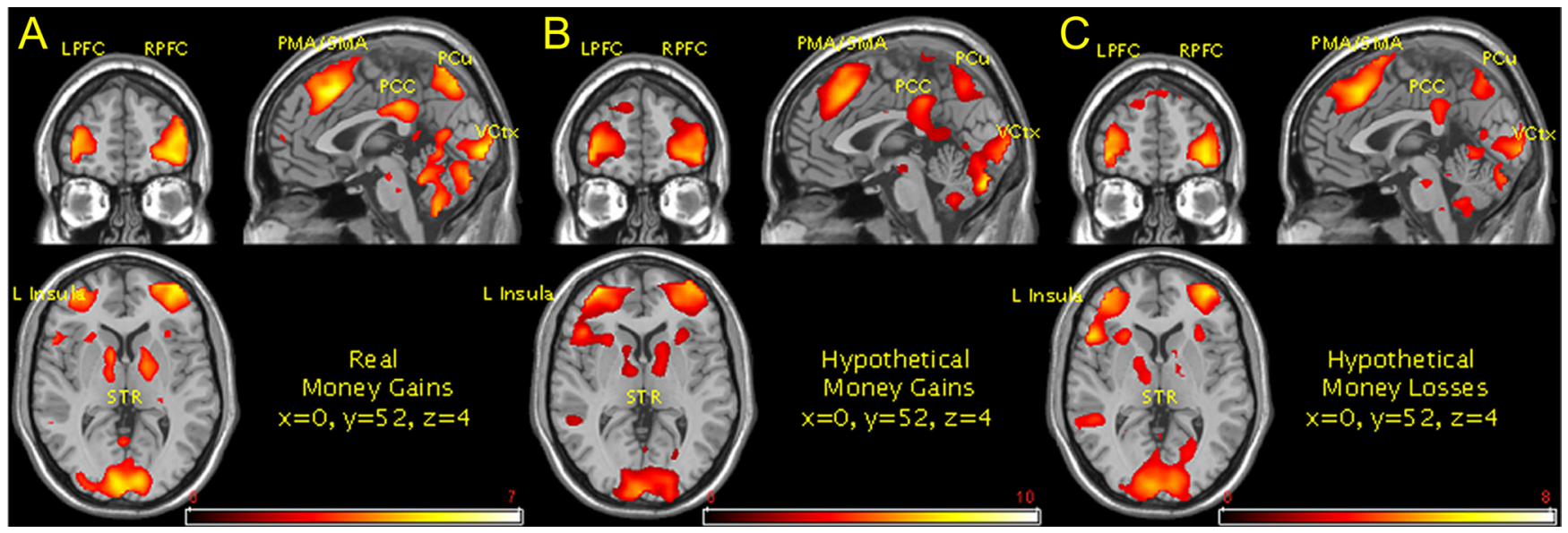

Figure 2. fMRI random effects results for real money gains $(\boldsymbol{A})$, hypothetical money gains $(\boldsymbol{B})$, and hypothetical money losses $(\boldsymbol{C})$ discounting conditions, contrasting the discounting trials where individuals responded to their preference for smaller sooner or larger later rewards/losses, with control events where participants responded to their preference for gaining or losing dollar amounts where the temporal delay component has been removed (FDR: $p<0.05$ ). Resulting areas of signal change occurred in similar regions across all three conditions: left and right lateral prefrontal cortex (LPFC/RPFC), PMA and SMA, posterior cingulate cortex (PCC), bilateral insula (seen lateralized primarily to the left hemisphere above), precuneus (PCu), visual cortex (VCtx), striatum (STR), and left and right parietal (data not shown)

counting behavior between behavioral and scanning sessions. Individual's respective $\ln (k)$ values (as estimated from behavioral session data) were found to be positively and significantly correlated with the ratio of preference for immediate amounts in both the RMG $(r=+0.73, p<0.0001)$ and HMG $(r=+0.52, p=$ 0.003 ) conditions, indicating that participant's with high rates of discounting showed a greater preference for immediate gains (real and hypothetical) in the scanning sessions. Fisher's $z$ transformation was used to test for difference in effect size between the two correlation coefficients from the gains conditions and indicated no significant difference between the measures, $Z=1.345$, $p=0.09$. Consistent with theory, high rates of the discounting of losses were associated with preference for delayed losses in the HML condition, although this relationship did not meet statistical significance $(r=+0.30, p=0.15)$. Participant responses in the RMG condition were significantly correlated with responses in the HMG condition $(r=+0.74, p<0.0001)$, whereas HML responses were inversely correlated with those in both gains conditions (HMG: $r=-0.42, p<0.0001$; RMG: $r=-0.42, p<$ $0.0001)$, signifying a parallel between increasing ratio of preference for immediate gains and a decreasing ratio preference for immediate losses. On average, participant's responses to replicated discounting trials were consistent with theory between the two gains conditions $87.0 \%$ ( $\pm 3.3 \%$ ) of the time, between HML and RMG $(74.3 \pm 6.3 \%)$, and HML and HMG (75.9 $\pm 4.8 \%)$. Behavior across these analyses was widely consistent withinsubject and also with discounting theory.

Mean response time in the discounting trials of the RMG condition (2360 $\pm 53 \mathrm{~ms}$ ) was not significantly different from that in the HMG condition $(2396 \pm 57 \mathrm{~ms})(t=0.83, p=0.40)$, whereas HML mean response time $(2540 \pm 67 \mathrm{~ms})$ was significantly greater than both the RMG $(t=-4.26, p<0.0001)$ and HMG $(t=-3.41, p=0.0007)$ conditions, perhaps indicating increased deliberation in selecting a preferred loss as opposed to a gain.

\section{Imaging results}

An initial conjunction analysis of the trial types (discount and control) in all three conditions was performed at the second level to assess areas of significant overlap between the two trial types to ensure that contrasting, discounting, and control tri- als would not inadvertently annul signal change in brain regions other than those related to rudimentary task performance (e.g., visual processing, motor activity). Results at an uncorrected threshold $(p<0.001)$ revealed that no areas other than those intended would be canceled out in a contrast of the two trial types (supplemental Fig. S1, available at www. jneurosci.org as supplemental material). Random effects analysis of responses to discounting trials versus control trials resulted in significant areas of signal change using an FDR threshold of $p<0.05$ for contrasts in RMG, HMG, and HML conditions (Fig. 2A-C, respectively) (see supplemental Figs. S2-S4, and full listing of activations in supplemental Tables S2-S4, available at www.jneurosci.org as supplemental material). The resulting areas of signal change across all conditions included areas previously identified in imaging studies investigating the neural correlates of decision making in delay discounting (McClure et al., 2004, 2007; Boettiger et al., 2007; Monterosso et al., 2007; Wittmann et al., 2007). These include areas of executive functioning (i.e., lateral prefrontal cortex, parietal cortex) and limbic areas (i.e., striatum, posterior cingulate), with bilateral striatum reaching statistical threshold in RMG and HMG conditions but lateralizing primarily to the left hemisphere in the HML condition. Significant signal change within the limbic areas including the striatum have been previously reported and have been indirectly shown to be positively correlated with individual's level of discounting (Hariri et al., 2006). Signal change in premotor and supplementary motor areas have been noted in previous studies but were regarded as part of rudimentary task performance (i.e., pushing a button representing the preferred choice). However, we note the same areas of significant signal change in all discounting conditions where the contrasting control condition also required response. This contrast theoretically should eliminate any signal change associated with the motor (preand supplementary) requirements of task performance, suggesting that signal change within premotor area (PMA) and supplementary motor area (SMA) is indicative of another task-associated process, perhaps cognitive in nature. Signal change within the bilateral anterior insula was identified in all three conditions and has been previously shown to correlate behaviorally with the delay of gratification in a delay- 

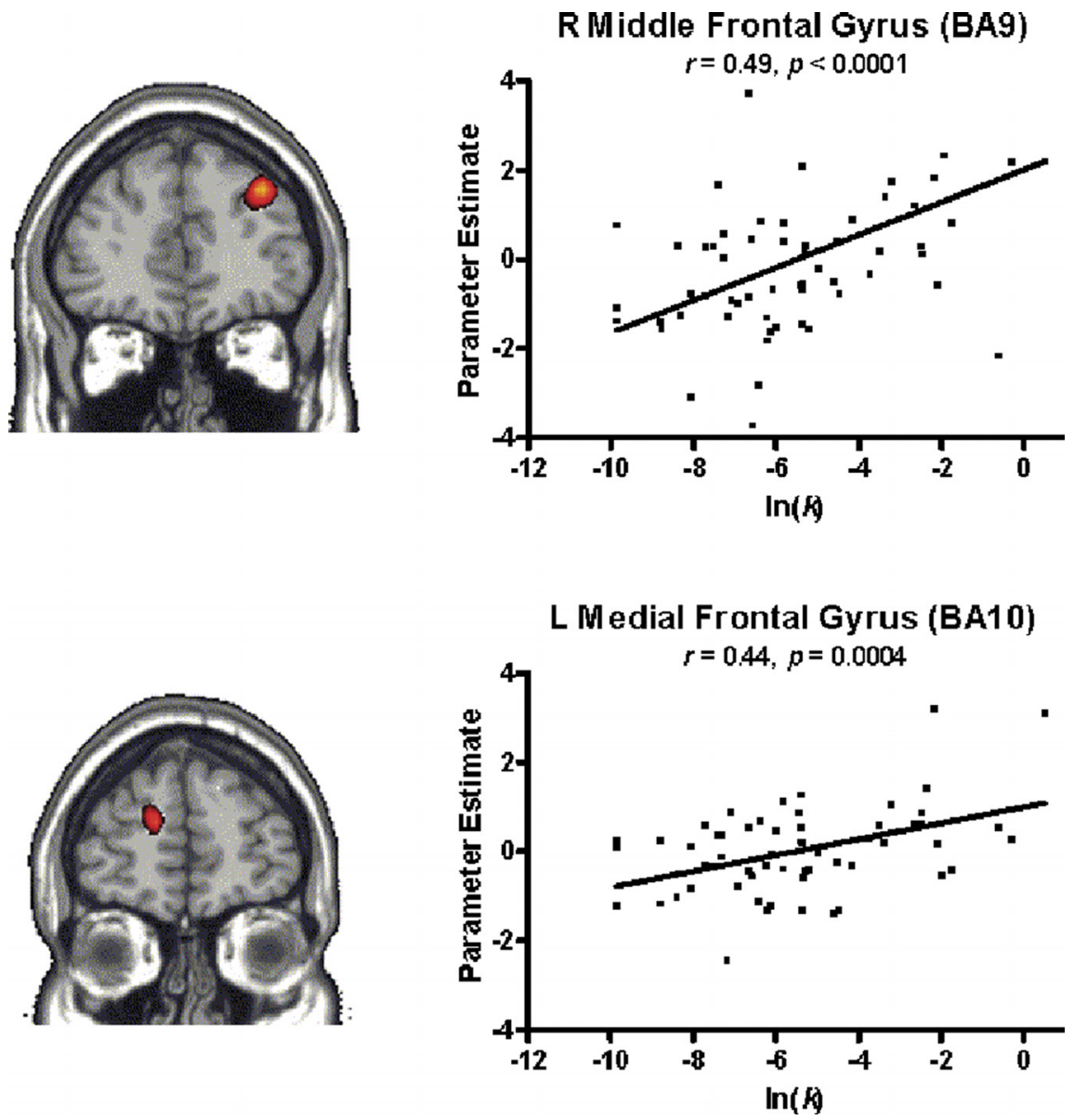

Figure 3. fMRI correlation analysis of random effects contrasts (discounting trials $>$ control trials) in combined RMG and HMG conditions. Log transformed $k$ values $(\ln (k))$ estimated from data collected before scanning used to predict areas correlated with discounting across subjects. Increased activity in the right middle frontal gyrus, BA9 $(\boldsymbol{A})$, and left medial frontal gyrus, BA10 $(\boldsymbol{B})$, are both shown here to be significantly correlated with increasing levels of discounting using an uncorrected threshold of $p<0.001$ and an extent threshold of five contiguous voxels. Results are displayed at $p<0.005$. Additional areas of significant correlation are listed in Table 1.

discounting procedure. Precuneus area of activation noted here in all conditions have also been revealed in previous studies and is known to have extensive afferent and efferent connections with both cortical (lateral parietal, frontal, anterior cingulate) and subcortical (thalamus, striatum, brainstem) areas (Cavanna and Trimble, 2006). Behavioral correlates associated with the precuneus include motor coordination associated with visuo-spatial imagery (Wenderoth et al., 2005), episodic memory retrieval (Lundstrom et al., 2005), and selfprocessing (Kircher et al., 2002; Kjaer et al., 2002). Signal change within the V2 area of the visual cortex-Brodmann's area 18 (BA18) - is also shown to be significant across all conditions within the discounting more than control contrast but may be explained by either the increased visual information presented in the discounting trials compared with the control trials, the resulting latency in stimulus presentation (with regard to response) of the discounting trials compared with the control trials, or a combination of both.

To detect whether the neural correlates of the decisionmaking process across theses three discounting conditions would elicit different levels of activation and/or different anatomical brain regions, random effects results from each of the independently analyzed discounting conditions were subsequently used in a repeated-measures ANOVA to test for main effect differences across discounting conditions. After controlling for multiple comparisons using an FDR threshold $(p<0.05)$, no differences in signal change were detected, inferring congruence of neural activity regardless of commodity or sign. We were, however, able to distinguish limited differences in paired $t$ tests when we did not control for multiple comparisons using an uncorrected threshold of $p<0.001$ and an extent threshold of five contiguous voxels (see supplemental materials, available at www.jneurosci.org as supplemental material, for figures and tables of differences between conditions). In the gains conditions comparison, $\mathrm{RMG}>\mathrm{HMG}$ resulted in a single cluster ( $n=6$ contiguous voxels) located in the left locus coeruleus, a structure outside of the cognitive and limbic areas typically associated with the deliberative process, but implicated in the processing of salient sensory information (Berridge and Waterhouse, 2003). HMG > RMG resulted in loci in the right frontal gyrus (BA48) $(n=13)$, left middle frontal gyri (BA9) $(n=6)$, and the right inferior parietal lobule (BA40) $(n=$ 6). Comparison of the hypothetical conditions, HMG $>$ HML resulted in no differential signal change above threshold, whereas HML > HMG comparison identified signal change in the left superior frontal gyrus (BA6) $(n=20)$, left superior temporal gyrus $(\mathrm{BA} 38)(n=6)$, and the right occipital lobe, precuneus (BA31) $(n=6)$. RMG $>$ HML contrast identifies a single locus of signal change in the left cerebellum, inferior semilunar lobule $(n=7)$, and HML $>$ RMG resulted in five areas above threshold; left occipital lobe (cuneus) $(n=13)$, left middle frontal gyrus (BA47) $(n=28)$, right occipital lobe (cuneus) $(n=5)$, right frontal lobe (BA9) $(n=7)$, and left medial frontal gyrus (BA8) $(n=9)$.

Given the strong correlation between participants' discounting behavior measured before scanning and their preference for immediate reward in both the real and hypothetical gains conditions within the scanner, we conducted a simple correlation analysis using imaging data from both RMG and HMG conditions with prescan $\ln (k)$ values as predictors, to identify regions associated with increasing levels of discounting. Using an uncorrected threshold of $p<0.001$ and an extent threshold of five contiguous voxels, two areas within the dorsal prefrontal cortex correlated highly with log transformed $k$ values: right middle frontal gyrus (BA9) and left medial frontal gyrus (BA10) (Fig. 3). Additional areas within the cerebellum and cuneus were also identified (Table 1). The dorsal lateral prefrontal cortex has been identified previously in the functional imaging of intertemporal choice and correlated with behavioral measures of impulsivity (Boettiger et al., 2007; Hoffman et al., 2008), whereas McClure et al. (2004, 2007) 
identified these executive functioning areas as being uniformly engaged without respect to delay.

\section{Discussion}

In this study, we investigate the similarities and disparities of delay discounting of real and hypothetical rewards and hypothetical loses, within both a behavioral and neuroimaging framework. Based on previous behavioral results, we hypothesized that (1) there would be no statistical within-subject difference in the estimated measure of discounting between equal amounts of a real and hypothetical gains (Johnson and Bickel, 2002; Madden et al., 2003, 2004; Lagorio and Madden, 2005) and (2) losses of hypothetical money would be discounted less than gains (Murphy et al., 2001; Estle et al., 2006; Yi et al., 2006). The first hypothesis was confirmed, with no within-subject difference observed in the discounting of real and hypothetical money gains. The second hypothesis was not confirmed, with no significant within-subject difference in the levels of discounting of gains and hypothetical money losses detected (Fig. 2A,B).

Based on previous neuroimaging results, we hypothesized that detection of fMRI signal change in limbic areas associated with reward would be greater when deliberating real money outcomes compared with hypothetical ones with no difference in the levels of executive functioning areas. However, we were uncertain as to the extent these areas of activity would generalize within the losses condition. Results from the independent random effects analysis of each condition produced robust activations in limbic and executive functioning areas across all three discounting conditions. However, no areas within the limbic system survived repeated-measures analysis either at a corrected (FDR: $p<0.05$ ) or uncorrected threshold $(p<0.001)$. These findings challenge the notion of greater limbic response to real reward outcomes compared with hypothetical ones based on a hedonic reaction to the former in contrast to the latter. We found significant levels of activation within striatum and posterior cingulate in conditions with both real and hypothetical rewards as well as hypothetical losses, again with no differences in activation levels when these conditions are directly compared. Areas of activation within the PMA and SMA noted here have also been noted in previous neuroimaging studies of delay discounting but have been attributed to motor-related requirements (i.e., button push). However, our experimental design incorporated control trials that included a button push requirement to offset an identical requirement in the contrasting discounting trials. Significant activation in these areas (PMA/SMA) may suggest another functional role in the deliberative process of delay discounting.

Activation within the medial prefrontal cortex has been associated with the availability of an immediate reward outcome (McClure et al., 2004, 2007), whereas a significant correlation between activation of the medial prefrontal/anterior cingulate cortex (BA32/24) with subjective value was shown to be independent of immediacy of reward (Kable and Glimcher, 2007). Our imaging results did not reveal activation within this region although, or perhaps attributable to, the presence of an immediate reward/loss option in all discounting trials. Results from the respective repeated-measures analyses of fMRI data here do not support the hypothesis of significantly different levels of activation within limbic areas in association with valence (gain vs loss).
We further hypothesized, based on previous findings, that areas associated with executive functioning (lateral prefrontal and parietal cortex) would not vary significantly in the level of signal change between the decision-making processes related to preference of real/hypothetical rewards or gains/losses. Here, the results are inconclusive. Although statistical significance was not reached after controlling for multiple comparisons, uncorrected signal change differences were detected in the middle and medial frontal lobes in contrasts comparing both hypothetical outcomes to real money gains. Whether these differences in the BOLD signal are indicative of a difference in the cognitive-processing requirements between the respective conditions is unclear. Given that analysis of the prescan behavioral data indicated no withinsubject differences in the level of discounting across the three conditions, it would seem unlikely that the differences noted in the fMRI data would have a substantial influence on the process overall.

The correlation between behavioral estimates and imaging data in the gains conditions revealed areas within the left medial frontal (BA10) and right middle frontal (BA9) gyri, indicating increased activity associated with increasing $\ln (k)$ values. Areas within the prefrontal cortex (PFC) have been identified with behavioral measures in previous studies of delay discounting. Monterosso et al. (2007) found increased signal change in the right ventrolateral prefrontal cortex (VLPFC) to be associated with less discounting, McClure et al. (2004) found both right dorsolateral prefrontal cortex and right VLPFC responded at a significantly higher level in response to hard choices compared with easy. Both Boettiger et al. (2007) and Hoffman et al. (2008), using hypothetical money gains of $\$ 100$ money, reported a positive correlation between delay-discounting measures of impulsivity and increased BOLD signal in the left dorsal PFC and right superior frontal gyrus (BA9), respectively. Although our results directly support those of Boettiger et al. (2007) and Hoffman et al. (2008), we cannot dismiss the possibility that responding to the discounting trials within our study was more difficult for those participants with higher levels of discounting. However, where McClure et al. (2004) used response times to substantiate hard from easy choices, we were unable to significantly correlate participant's average response times with $\ln (k)$ values here $(r=-0.1, p=0.26)$, suggesting that difficulty in the deliberation of discounting trials was not associated with level of discounting. One plausible explanation for the similarities between findings here and with those of Boettiger et al. (2007) and Hoffman et al. (2008) is the utilization of comparable control conditions within the experimental designs. In all three experiments, control trials were used in which a deliberative response was required and subsequently contrasted with discounting trials to form the random effects results used in the respective correlation analyses. Although control trials in our design were replications of the stimuli presented in the 
discounting trials without explicit reference to a temporal component, Boettiger et al. (2007) used control trials that prompted the subject to choose either the smaller or the larger amount of two alternatives requiring the subject to ignore the explicit temporal component. Hoffman et al. (2008) contrasted discounting trials with control trials whereby subjects responded to their preference between two choices in which either the delay or reward was held constant (e.g., $\$ 50$ today vs $\$ 50$ in $65 \mathrm{~d}, \$ 50$ in $12 \mathrm{~d}$ vs $\$ 75$ in $12 \mathrm{~d}$ ). Our results differ from those of Monterosso et al. (2007) who used a contrasting effect in their correlation analyses of "hard choice versus no choice" where "no choice" trials simply required the subject to identify the location of a single option presented on either the left or right side of the screen, thereby requiring no assessment of the stimuli other than spatial location.

A potential limitation or confound to this study is the plausibility of participants' adoption of rule-governed behavior in lieu of an authentic intertemporal choice deliberative process given exposure to discounting examinations in the prescan behavioral session. Given the degree of concordance of these neuroimaging findings with previously published works, we believe that the neural correlates as identified here reflect the nature of the discounting decision-making process as intended although lack of activity in the medial orbital frontal cortex and anterior cingulate regions as identified in previous studies admittedly gives us pause. The behavioral and imaging results from this study suggest that participant's undergoing delay-discounting conditions discount the value of a real money outcome equal to that of a hypothetical money outcome and that the neural correlates of these comparable conditions do not differ to a great degree within the primary brain regions believed to be associated with this cognitive process (limbic and executive). Whether a detectable behavioral difference between the discounting of these three conditions would ultimately provide significant difference within the imaging data is plausible, but previous studies comparing differing levels of discounting between groups have failed to yield any noteworthy findings.

The value of the results of this study are at least twofold. First, the results are practical in that they indicate that real and hypothetical outcomes not only produce comparable behavioral outcomes but also comparable neural correlates. This means that the study of hypothetical outcomes are not questionable a priori and may very well be consistent with real outcomes if it were feasible or ethical to evoke real outcomes in research studies. Second, and more importantly, these results suggest that the brain regions related to considering real and hypothetical future outcomes appear to be equivalent. Given that consideration of the future is an important neurobehavioral process, we show here that the process does not appear to change when the outcomes of those considerations are fictive or real. This makes sense from an evolutionary basis as humans often have to consider future outcomes to make plans important to survival. It would be difficult to imagine evolutionary pressure that would result in differing neurobehavioral processes for future events that occur versus those that do not.

\section{References}

Ainslie G, Haendel V (1983) The motives of the will. In: Etiology aspects of alcohol and drug abuse (Gottheil E, Druley K, Skodola T, Waxman H, eds). Springfield, IL: Charles C. Thomas.

Baker F, Johnson MW, Bickel WK (2003) Delay discounting in current and never-before cigarette smokers: similarities and differences across commodity, sign, and magnitude. J Abnorm Psychol 112:382-392.
Berridge CW, Waterhouse BD (2003) The locus coeruleus-noradrenergic system: modulation of behavioral state and state-dependent cognitive processes. Brain Res Brain Res Rev 42:33-84.

Bickel WK, Johnson MW (2003) Junk time: pathological behavior as the interaction of evolutionary and cultural forces. In: Choice, behavioral economics and addictions (Heather N, Vuchinich R, eds), pp 249-271. Amsterdam: Pergamon.

Bickel WK, Marsch LA (2001) Toward a behavioral economic understanding of drug dependence: delay discounting processes. Addiction 96:73-86.

Boettiger CA, Mitchell JM, Tavares VC, Robertson M, Joslyn G, D’Esposito M, Fields HL (2007) Immediate reward bias in humans: fronto-parietal networks and a role for the catechol-O-methyltransferase $158(\mathrm{Val} / \mathrm{Val})$ genotype. J Neurosci 27:14383-14391.

Cavanna AE, Trimble MR (2006) The precuneus: a review of its functional anatomy and behavioural correlates. Brain 129:564-583.

Charlton SR, Fantino E (2008) Commodity specific rates of temporal discounting: does metabolic function underlie differences in rates of discounting? Behav Processes 77:334-342.

Coffey SF, Gudleski GD, Saladin ME, Brady KT (2003) Impulsivity and rapid discounting of delayed hypothetical rewards in cocaine-dependent individuals. Exp Clin Psychopharmacol 11:18-25.

Du W, Green L, Myerson J (2002) Cross-cultural comparisons of discounting delayed and probablistic rewards. Psychol Rec 52:479-492.

Estle SJ, Green L, Myerson J, Holt DD (2006) Differential effects of amount on temporal and probability discounting of gains and losses. Mem Cognit 34:914-928.

Estle SJ, Green L, Myerson J, Holt DD (2007) Discounting of monetary and directly consumable rewards. Psychol Sci 18:58-63.

Hariri AR, Brown SM, Williamson DE, Flory JD, de Wit H, Manuck SB (2006) Preference for immediate over delayed rewards is associated with magnitude of ventral striatal activity. J Neurosci 26:13213-13217.

Hoffman WF, Schwartz DL, Huckans MS, McFarland BH, Meiri G, Stevens AA, Mitchell SH (2008) Cortical activation during delay discounting in abstinent methamphetamine dependent individuals. Psychopharmacology (Berl) 201:183-193.

Johnson MW, Bickel WK (2002) Within-subject comparison of real and hypothetical money rewards in delay discounting. J Exp Anal Behav 77:129-146.

Kable JW, Glimcher PW (2007) The neural correlates of subjective value during intertemporal choice. Nat Neurosci 10:1625-1633.

Kirby KN, Petry NM, Bickel WK (1999) Heroin addicts have higher discount rates for delayed rewards than non-drug-using controls. J Exp Psychol Gen 128:78-87.

Kircher TT, Brammer M, Bullmore E, Simmons A, Bartels M, David AS (2002) The neural correlates of intentional and incidental self processing. Neuropsychologia 40:683-692.

Kjaer TW, Nowak M, Lou HC (2002) Reflective self-awareness and conscious states: PET evidence for a common midline parietofrontal core. Neuroimage 17:1080-1086.

Lagorio CH, Madden GJ (2005) Delay discounting of real and hypothetical rewards III: steady-state assessments, forced-choice trials, and all real rewards. Behav Processes 69:173-187.

Lundstrom BN, Ingvar M, Petersson KM (2005) The role of precuneus and left inferior frontal cortex during source memory episodic retrieval. Neuroimage 27:824-834.

Madden GJ, Petry NM, Badger GJ, Bickel WK (1997) Impulsive and selfcontrol choices in opioid-dependent patients and non-drug-using control participants: drug and monetary rewards. Exp Clin Psychopharmacol $5: 256-262$.

Madden GJ, Begotka AM, Raiff BR, Kastern LL (2003) Delay discounting of real and hypothetical rewards. Exp Clin Psychopharmacol 11:139-145.

Madden GJ, Raiff BR, Lagorio CH, Begotka AM, Mueller AM, Hehli DJ, Wegener AA (2004) Delay discounting of potentially real and hypothetical rewards: II. Between- and within-subject comparisons. Exp Clin Psychopharmacol 12:251-261.

Mazur JE (1987) An adjusting procedurefor studying delayed reinforcement. In: Quantitative analysis of behavior (Commons ML, Mazur JE, eds), pp 55-73. Hillsdale, NJ: Erlbaum.

McClure SM, Laibson DI, Loewenstein G, Cohen JD (2004) Separate neural 
systems value immediate and delayed monetary rewards. Science 306:503-507.

McClure SM, Ericson KM, Laibson DI, Loewenstein G, Cohen JD (2007) Time discounting for primary rewards. J Neurosci 27:5796-5804.

Monterosso JR, Ainslie G, Xu J, Cordova X, Domier CP, London ED (2007) Frontoparietal cortical activity of methamphetamine-dependent and comparison subjects performing a delay discounting task. Hum Brain Mapp 28:383-393.

Murphy JG, Vuchinich RE, Simpson CA (2001) Delayed reward and cost discounting. Psychol Rec 51:571-588.

Myerson J, Green L, Warusawitharana M (2001) Area under the curve as a measure of discounting. J Exp Anal Behav 76:235-243.

Odum AL, Rainaud CP (2003) Discounting of delayed hypothetical money, alcohol, and food. Behav Processes 64:305-313.

Ohmura Y, Takahashi T, Kitamura N, Wehr P (2006) Three-month stability of delay and probability discounting measures. Exp Clin Psychopharmacol 14:318-328.

Takahashi T, Furukawa A, Miyakawa T, Maesato H, Higuchi S (2007) Two-month stability of hyperbolic discount rates for delayed monetary gains in abstinent inpatient alcoholics. Neuro Endocrinol Lett 28:131-136.

Wenderoth N, Debaere F, Sunaert S, Swinnen SP (2005) The role of anterior cingulate cortex and precuneus in the coordination of motor behaviour. Eur J Neurosci 22:235-246.

Wittmann M, Leland DS, Paulus MP (2007) Time and decision making: differential contribution of the posterior insular cortex and the striatum during a delay discounting task. Exp Brain Res 179: 643-653.

Yi R, Gatchalian KM, Bickel WK (2006) Discounting of past outcomes. Exp Clin Psychopharmacol 14:311-317. 